Images dans le monde ibérique et ibéricoaméricain

$8 \mid 2015$

L'Histoire et ses récits entre images, fictions et paratextes

\title{
Une traduction castillane anonyme de l'Éthique à Nicomaque dans la bibliothèque du Marquis de Santillane (ms BNE 10268)
}

\section{Mélanie Jecker}

\section{OpenEdition}

Journals

Édition électronique

URL : http://journals.openedition.org/agedor/425

DOI : 10.4000 /agedor.425

ISSN : 2104-3353

Éditeur

Laboratoire LISAA

Référence électronique

Mélanie Jecker, « Une traduction castillane anonyme de l'Éthique à Nicomaque dans la bibliothèque du Marquis de Santillane (ms BNE 10268) ", L'Âge d'or [En ligne], 8 | 2015, mis en ligne le 01 février 2016, consulté le 01 mai 2019. URL : http://journals.openedition.org/agedor/425; DOI : 10.4000/agedor.425 


\title{
Mélanie JECKER
}

Université de Paris-Sorbonne, équipe d'accueil CLEA

(Civilisations et Littératures de l'Espagne et de l'Amérique du Moyen Âge aux Lumières)

\section{Une traduction castillane anonyme de l'Éthique à Nicomaque dans la bibliothèque du Marquis de Santillane (ms BNE 10268)}

\begin{abstract}
Résumé : Cet article étudie deux types d'inscriptions marginales dans le manuscrit 10268 de la Biblioteca Nacional de España, manuscrit du XVe siècle ayant appartenu à la bibliothèque du Marquis de Santillane et contenant une traduction castillane de l'Éthique à Nicomaque d'Aristote: j'analyse d'une part le rapport entre le texte traduit et les annotations marginales qui l'accompagnent, d'autre part les citations éparses copiées sur les premiers et derniers feuillets du manuscrit, afin de mettre en évidence l'importance qu'y revêt le thème de la prudence, comme vertu morale mais aussi et surtout comme vertu politique.
\end{abstract}

Mots-clés: Éthique à Nicomaque - traduction - annotations marginales - Marquis de Santillane bibliothèque - prudence - conseil

Resumen: Este artículo estudia dos tipos de inscripciones marginales en el manuscrito 10268 de la Biblioteca Nacional de España, manuscrito del siglo XV que perteneció a la biblioteca del Marqués de Santillana y que contiene una traducción castellana de la Ética a Nicómaco de Aristóteles : analizo por una parte la relación entre el texto traducido y las notas marginales que lo acompañan, por otra parte las citas dispersas copiadas en los primeros y últimos folios del manuscrito, para poner de manifiesto la importancia atribuida al tema de la prudencia, como virtud moral pero también y sobre todo como virtud política.

Palabras clave : Ética a Nicómaco - traducción - notas marginales - Marqués de Santillana - biblioteca prudencia - consejo

\section{Introduction}

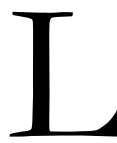

a riche bibliothèque du Marquis de Santillane réunit diverses formes de vulgarisation de la doctrine éthique aristotélicienne, depuis la traduction castillane du texte intégral de l'Éthique à Nicomaque (manuscrit 10268 de la Bibliothèque Nationale d'Espagne) jusqu'à sa lecture humaniste par Matteo Palmieri dans son Libro della vita civile (manuscrit BNE Res/244), en passant par une table des termes renvoyant aux principaux concepts de l'Éthique par Thomas d'Aquin - manuscrit BNE 10269 - et par trois compendia de l'Éthique, l'un en latin - la Summa de virtutibus de Guido Vernani da Rimini, manuscrit BNE 10269 -, un autre en castillan - la traduction de l'Isagogicon moralis disciplinae de Leonardo Bruni, manuscrit BNE 10212 -, le troisième en italien - la traduction italienne de la Summa Alexandrinorum par Taddeo Alderotti, manuscrit BNE 10124. 
Le marquis possédait donc non seulement le texte complet de l'Éthique, mais aussi ses interprétations médiévales, scolastiques comme la Tabula de Thomas d'Aquin aussi bien qu'humanistes, comme la Vita civile de Palmieri. Ces diverses formes de transmission de l'Éthique aristotélicienne illustrent différents aspects du processus de réception de la doctrine éthique du Stagirite. Parmi elles, la traduction castillane du manuscrit 10268 revêt un intérêt particulier car, étant la traduction du texte intégral d'Aristote et non une paraphrase ou un résumé, elle met en lumière, notamment dans le rapport entre le texte central et ses annotations marginales, les difficultés que supposa la réception d'une doctrine complexe.

Le manuscrit 10268 contient la traduction castillane de l'Éthique, suivie de celle de l'Économique à partir du folio 213 ; il fut copié par un certain Morillo dont le nom apparait au folio $230 \mathrm{v}$. Les quelques feuillets initiaux et finaux du manuscrit, qui précèdent et suivent les deux traductions, sont couverts de notes éparses et de citations d'auctoritates diverses, Aristote principalement, en latin et en castillan, écrites de plusieurs mains. Mario Schiff ne signale pas ce manuscrit parmi les livres ayant de façon certaine appartenu au Marquis, mais le fait néanmoins figurer dans son étude de la célèbre bibliothèque, considérant donc que sa copie est antérieure à la mort du Marquis en $1458^{1}$.

Or cette traduction, bien qu'il s'agisse probablement de la première traduction castillane de l'Éthique d'Aristote, est très rarement mentionnée dans les travaux de recherche portant sur les traductions médiévales, et quand elle l'est, elle ne fait l'objet d'aucune analyse approfondie, contrairement à la traduction du prince Carlos de Viana (1457) étudiée par Carlos Heusch ${ }^{2}$, ou au compendium castillan de l'Éthique réalisé en 1467 par l'humaniste sévillan Nuño de Guzmán, bien étudié par A. R. D Pagden et Peter Russell ${ }^{3}$, puis par Jeremy Lawrance ${ }^{4}$ et Salvador Cuenca i Almenar.

Parmi les ouvrages consacrés aux traductions médiévales réalisées dans la Péninsule ibérique, plusieurs ne mentionnent pas ce manuscrit. C'est le cas de Menéndez y Pelayo dans Biblioteca de traductores españoles ${ }^{6}$, mais aussi de Carlos Alvar qui, à propos des traductions castillanes de l'Éthique d'Aristote, attribue à Pedro Díaz de Toledo, chapelain du marquis de Santillane et auteur d'un Razonamiento en la muerte del marqués de Santillana, une traduction - perdue - de la Summa Alexandrinorum, traduction latine d'un résumé arabe de l'Éthique, qui n'a rien à voir avec notre manuscrit ${ }^{7}$. Dans Traducciones y traductores, Carlos Alvar mentionne à nouveau Pedro Díaz de Toledo comme traducteur de l'Éthique au service du Marquis de Santillane, et suggère qu'il traduisait directement depuis le grec, mais n’apporte pas plus de précisions sur cette hypothétique traduction, et ne mentionne pas le manuscrit de la $\mathrm{BNE}^{8}$.

Quant aux ouvrages qui le mentionnent, Mario Schiff n'en offre qu'une description sommaire sans apporter d'informations sur la traduction ni sur le traducteur'. De même, les

${ }^{1}$ SCHIFF, Mario, La bibliothèque du Marquis de Santillane, Paris, Librairie Émile Bouillon, 1905.

2 HEUSCH, Carlos, «La morale du prince Charles de Viana », in Atalaya, 4, 1993, p. 93-226.

${ }^{3}$ PAGDEN A. R. D et RUSSELL P., « Nueva luz sobre una versión española cuatrocentista de la Ética a Nicómaco: Bodleian Library, Ms Span D.1 ", in Homenaje a Guillermo Guastavino, Miscelánea de estudios en el año de su jubilación como Director de la Biblioteca Nacional, Madrid, Asociación Nacional de Bibliotecarios, Archiveros y arqueólogos, 1974, p. 126-146.

${ }^{4}$ LAWRANCE, Jeremy, "Nuño de Guzmán and early Spanish humanism : Some reconsiderations ", in Medium Aevum, 51, 1982, p. 55-84.

${ }^{5}$ CUENCA I ALMENAR, Salvador, «L'Ética nicomaquea d'Aristótil en un compendi català del segle XV », in Annuari de la Societat Catalana de Filosofia, 33, 2012, p. 7-119.

${ }^{6}$ MENÉNDEZ Y PELAYO, Marcelino, Biblioteca de traductores españoles, in Obras completas, Santander, CSIC, 1950, vol. 1 , p. 307.

7 ALVAR, Carlos, et LUCÍA MEGÍAS, José Manuel, Repertorio de traductores del siglo XV, Madrid, Ollero y Ramos, 2009, p. 76-82. En effet, dans le prologue à sa traduction des Proverbios de Sénèque, Díaz de Toledo affirme avoir également traduit «ciertas autoridades e notables de la philosophia moral de Aristótiles que fueron sacadas de la trasladación arábica en latín. »

${ }^{8}$ ALVAR, Carlos, Traducciones y traductores. Materiales para una historia de la traducción en Castilla durante la Edad Media, Alcalá de Henares, Centro de Estudios Cervantinos, 2010, p. 286.

${ }_{9}$ SCHIFF, Mario, La bibliothèque..., op. cit., p. 31-36. 
auteurs du catalogue de l'exposition sur la bibliothèque Mendoza-Infantado, comme Schiff avant eux, mentionnent le manuscrit sans astérisque, signalant ainsi qu'ils ne peuvent affirmer avec certitude qu'il ait appartenu au Marquis de son vivant, et n'offrent aucune précision supplémentaire $^{10}$. Plus récemment, Giuseppina Grespi décrit brièvement le manuscrit en indiquant simplement que le traducteur n'est probablement pas Alfonso de Cartagena ${ }^{11}$.

Plusieurs raisons peuvent expliquer ce désintérêt.

1 - La traduction est de fort piètre qualité, souvent fautive, voire incompréhensible. Ceci explique aussi qu'elle n'ait pas été imprimée, contrairement aux deux autres. De cette maladresse je donnerai quelques exemples.

À propos de la recherche de l'honneur, le traducteur commet un contresens ou du moins opte pour une formulation ambiguë : «otros mas nobles eligieron el honor por ser casi fin de la vida politica [...] procuran el honor de los prudentes $»^{12}$. Le sens du texte d'Aristote est que ceux qui sont en quête d'honneurs souhaitent que les hommes prudents les jugent vertueux, mais la traduction suggère qu'ils souhaitent obtenir le même honneur que les prudents.

Au livre $\mathrm{X}$ apparaît une autre erreur, à propos du plaisir que chacun trouve à exercer l'activité qui lui est propre. À propos du plaisir que le sage trouve dans la spéculation, le texte latin de l'Éthique, dans la traduction de Robert Grosseteste (1246), explique «amator autem discipline mente circa theoremata, sic autem et reliquorum unusquisque ${ }^{13}$, et dans la traduction de Leonardo Bruni (1417), «Disciplinarum studio dediti intellectu circa prospicientiam rerum, eodem modo reliquorum singuli $»^{14}$, tandis que notre traducteur ajoute inopportunément l'idée de prudence, «el que la prudençia ama pone su entendimiento en las cosas especulativas ${ }^{15}$ : il commet ainsi un grave contresens, puisqu'Aristote distingue clairement la prudence, vertu intellectuelle s'appliquant à l'action en ce bas monde soumis à la contingence, et la sagesse par laquelle l'homme s'adonne à la spéculation, et qui porte sur des objets nécessaires, immuables et éternels. Peut-être a-t-il noté que l'expression « discipline mente » de Grosseteste évoquait la « recta ratio » qui dans l'Éthique désigne la vertu de prudence.

À propos du bonheur, Aristote explique que pour savoir ce qu'est le bonheur de l'homme, il faut déterminer quel est l'office propre de l'homme, mais la traduction castillane de cette idée est absolument incompréhensible :

Esto sera fecho tomando la obra del omne [...] Entonçes quedara una obradera cuyo es el entender, lo qual en dos maneras sera, o por via de dotrina e ensenança o por si mesmo entendera. E conviene que digamos que esta obra es segund la yntellectiva que es la singular e esta conviene dezir. E si la obra del omne es la obra del alma con entender o non sin entender commo sea una cosa en genero esta obra e esta buena obra. ${ }^{16}$

Voici un autre exemple de formulations incongrues, dans les développements consacrés à la notion de choix, ici traduite par «deliberaçión» :

\footnotetext{
${ }^{10}$ Exposición de la biblioteca de los Mendoza del Infantado en el siglo XV, Madrid, Junta técnica de archivos, bibliotecas y museos, 1958.

11 GRESPI, Giuseppina, Traducciones castellanas de obras latinas e italianas contenidas en manuscritos del siglo XV en las bibliotecas de Madrid y El Escorial, Madrid, Biblioteca Nacional de España, 2004, p. 49.

12 BNE, Ms. 10268, fol. 5r.

13 ARISTÓTELES, Ethica Nicomachea, GAUTHIER René-Antoine (éd.), Bruxelles, Desclée de Brouwer, 1972, Aristoteles Latinus XXVI, 3, p. 354 et 571.

14 ARISTÓTELES, Ethica, Valence, 1473, sans foliotation.

15 BNE, Ms. 10268, folio 196v.

${ }^{16}$ Ibid., folio 8r-v.
} 
La deliberaçion paresçe manifiesta mente que sea voluntaria e que non sean una cosa mas lo voluntario es mas conprehendiente [...] Que non conprehende la deliberaçion a ninguna cosa yrraçional, mas la saña e la cobdiçia son conprehendientes. ${ }^{17}$

De même au livre X, la réfutation aristotélicienne des arguments de Platon contre le plaisir est très confuse. Ainsi, l'idée que le plaisir n'est pas un bien en soi, contrairement à la prudence : «Ca sera mas escogible con la prudençia que sera vida de deleyte mas de lo que sera sin ella. E sy en seyendo conpuesto es mejor, non es el deleyte bueno en si $»^{18}$.

Enfin, à propos des divers types de biens, Aristote attribue la prééminence aux biens de l'âme, mais le choix de l'adjectif «animal» rend peu claire la traduction castillane de cette idée : «Los bienes se parten a tres espeçias [...] E destos los mas singulares e mejores son los animales, ca los fechos e las obras animales en la obra ponemos los correspondientes a la anima ${ }^{19}$.

Ces quelques exemples illustrent la difficulté de la réception des thèses contenues dans l'Éthique, due à leur nouveauté et complexité d'une part, d'autre part au caractère dense et souvent obscur de la traduction latine de Robert Grosseteste.

2 - Le traducteur est anonyme, contrairement aux cas illustres du prince Charles de Viana et l'humaniste sévillan Nuño de Guzmán.

3 - Le destinataire est inconnu, tandis que la traduction de Charles de Viana est adressée à son oncle Ferdinand V, roi d'Aragon, et celle de Nuño de Guzmán au grand noble Juan de Guzmán, seigneur de La Algaba. La traduction du manuscrit 10268, pourtant, fut très vraisemblablement réalisée pour le Marquis de Santillane ou pour le roi de Castille Jean II.

4 - Surtout, cette médiocre traduction ne semble pas pouvoir illustrer le proto-humanisme de la première moitié du Cuatrocientos, ni confirmer le rôle du marquis de Santillane, possesseur du manuscrit, comme introducteur en Castille de l'humanisme italien, comme le suggérait déjà Mario Schiff, la bibliothèque de Guadalajara ayant été «l'une des premières à ouvrir ses armoires aux conquêtes de l'humanisme italien [...] Le modeste berceau des nouvelles idées que l'humanisme italien communiqua à l'Espagne $»^{20}$. En effet, contrairement à la traduction du prince de Viana, basée sur la nouvelle version latine de Leonardo Bruni, le texte latin à partir duquel fut réalisée notre traduction n'est autre que la translatio vetus de Robert Grosseteste (1246), tant vitupérée par Leonardo Bruni ${ }^{21}$, et par ses émules castillans Pedro de Osma et Fernando de Roa, comme illustrant une ignorance et un manque de finesse dans l'étude des textes anciens, propres à un âge médiéval heureusement révolu.

J'apporterai ici quelques éléments suggérant que le texte latin ici traduit en castillan est la version de Robert Grosseteste.

À propos du juste milieu en quoi consiste la vertu morale, Aristote précise qu'il n'existe pas partout, puisque certains actes, comme le meurtre ou l'adultère, sont toujours mauvais ${ }^{22}$. On ne trouve pas ici l'ajout de Leonardo Bruni, «Et eodem modo dicendum est de injuria et ignavia et luxuria », traduit comme suit par Carlos de Viana «E por la semeiante manera es de dezir de la iniuria e necedat e luxuria $»^{23}$.

\footnotetext{
${ }^{17}$ Ibid., folio $37 \mathrm{v}$.

${ }^{18}$ Ibid., folio $190 \mathrm{v}$.

19 Ibid., folio 9v.

${ }^{20}$ SCHIFF, Mario, La bibliothèque ..., op. cit., p. LXXXIII-LXXXVI.

${ }^{21}$ Ceci en des termes fort sévères, "supinae crassaeque ruditatis », "ferreum hominem », "inermis et rudis ». Voir GONZÁLEZ-ROLÁN, T., MORENO HERNÁNDEZ, A., et SAQUERO SUÁREZ-SOMONTE, P., Humanismo y teoría de la traducción en España e Italia en la primera mitad del siglo XV, Madrid, Ediciones Clásicas, 2000, p. 178, 182 et 192.

22 BNE, Ms. 10268, folio 27v.

${ }^{23}$ La philosophia moral del Aristoteles, imprimée en 1509 à Saragosse, folio $61 \mathrm{r}$.
} 
À propos du courage politique, une annotation marginale résume: "primero pone la fortaleza politica de la qual son tres modos $»^{24}$, or c'est Robert Grosseteste qui utilise l'adjectif " politica », tandis que Leonardo Bruni choisit « civilis ».

De même, la notion de délibération, traduite par «consilium» chez Grosseteste et par « consultatio » chez Bruni, est ici rendue par « consejo ».

Enfin, la définition de la prudence, «Queda seer la prudençia abito verdadero hazedor con entender çerca de los bienes del omne e de los males $»^{25}$, est plus proche de la formulation de Grosseteste, «Relinquitur ergo ipsam esse habitum verum cum ratione activum circa hominis bona et mala $»^{26}$, que de celle de Bruni, "Restat ergo ut prudentia sit habitus verus cum ratione in his agendis versatus que sunt homini bona et mala »: « del omne» traduit le génitif « hominis », et l'idée d'action associée comme épithète, « activus », à « habitus », est ici rendue par « hazedor ».

Or, en ce milieu du XV $\mathrm{XV}^{\mathrm{e}}$ siècle, la nouvelle traduction latine de l'humaniste Leonardo Bruni circulait depuis plusieurs années en Europe, où elle s'était diffusée avec une rapidité surprenante, comme Bruni lui-même le souligne, « simul atque edita est, sic arripuerunt homines, ut momento paene temporis non solum Italia voluminibus eius repleta sit, verum etiam ad extremas terrarum oras convolarit $»^{27}$. La nouvelle traduction avait très tôt gagné la péninsule Ibérique, où elle fut adoptée en 1457 par Pedro de Osma comme texte de référence pour les cours de philosophie morale de l'université de Salamanque. Or le texte de Bruni était accompagné d'un prologue où le traducteur blâmait l'ancienne traduction de Grosseteste, inélégante et obscure, et ses translittérations incompréhensibles de termes grecs, «eubulia », "synesis », " gnome», «eustochia», etc. Rigoureusement contemporaine de ce basculement, la traduction qui nous occupe, pourtant, pouvait apparaittre comme déjà dépassée.

Ces diverses raisons éclairent l'indifférence en laquelle elle a été tenue jusqu'ici. Elle me semble toutefois avoir été injustement négligée, aussi tâcherai-je de montrer que, quoique maladroite et fautive, elle présente un grand intérêt au regard de l'histoire de la philologie et des idées politiques : l'histoire de la philologie, car contrairement à Robert Grosseteste, le traducteur castillan a pris soin de traduire toutes les notions clés du texte aristotélicien par des mots castillans d'usage courant, éliminant donc les translittérations de termes grecs tant blâmées par Leonardo Bruni, lesquelles en revanche réapparaissent, comme nous le verrons, dans les annotations marginales qui accompagnent la traduction.

Dans le rapport entre celle-ci et les inscriptions marginales réside en effet le second et principal intérêt philologique de ce manuscrit. J'analyserai à son propos deux types de marges : d'une part les marges proprement dites, qui encadrent le texte traduit, d'autre part les feuillets initiaux et finaux semés de citations diverses, qui constituent les marges du manuscrit lui-même. L'analyse de ces deux types de marges révèle deux aspects de la réception de l'Éthique dans la Castille du Cuatrocientos: le rapport entre le texte central et les annotations marginales met en évidence les points de doctrine plus difficiles qui exigèrent un effort de reformulation, tandis que le vade mecum qui occupe les premiers et derniers feuillets révèle quelles notions de l'Éthique intéressaient davantage les lecteurs, membres de la haute noblesse, qui fréquentaient la bibliothèque de Guadalajara. Or la notion de prudence y apparaît non seulement comme l'une des notions les plus complexes de l'Éthique, mais aussi, et c'est là ce qui rend ce manuscrit précieux à l'historien des mentalités, comme un enjeu conceptuel clé dans l'affrontement de deux conceptions du pouvoir royal et de son exercice, sous les règnes tumultueux de Jean II et d'Henri IV de Castille.

\footnotetext{
24 BNE, Ms. 10268, folio 48v.

25 Ibid., folio $106 \mathrm{v}-107 \mathrm{r}$.

26 Aristoteles Latinus, op. cit., p. 257.

27 « nada más ver la luz, los hombres se hicieron con ella de tal forma que en poco tiempo no sólo Italia estaba llena de ejemplares, sino que también alcanzó los últimos confines de las tierras ", Humanismo y teoría de la traducción..., op. cit., p. 268-269.
} 


\section{La traduction castillane et ses annotations marginales}

Si le texte central et les annotations marginales semblent avoir été copiés de la même main, en revanche leur conception respective peut difficilement être attribuée à une même personne : dans certains cas, les deux textes se complètent et s'éclairent mutuellement, au bénéfice du lecteur, mais dans beaucoup d'autres, tous deux sont discordants et rendent malaisée la compréhension du texte. Quant aux corrections apportées à la traduction sous forme de biffures, elles pourraient être dues à l'auteur des annotations, ou à une troisième personne, comme je le suggérerai plus loin.

\section{Les annotations éclairent le corps du texte}

Les inscriptions marginales offrent souvent un résumé limpide de développements traduits très confusément dans le texte principal. J'en donnerai quelques exemples.

Voici le résumé du chapitre VII du livre I : «aqui muestra que las obras segund virtud non solamente son delectables mas tanbien pulcras e buenas $»^{28}$.

Au livre II, plusieurs formulations synthétiques aident à comprendre le rôle du législateur, qui par ses lois rend vertueux les habitants de la cité, en leur imprimant de bons habitus. En face du texte central,

E ansi los que fazen las justiçias se fazen justos e los que la tenperança tenperados los que la fortaleza fuertes. Da fee de aquesto lo que se faze en las çibdades, que los que ordenan los fueros e leyes fazen acostunbrar a los moradores de la çibdad que sean buenos, e aquesta es la entinçion de qualquiera leyes ${ }^{29}$,

apparaissent les annotations «aqui manifiesta lo que ha dicho por señal, que obrando nos fazemos virtuosos", "aqui muestra que las obras sean que causen la virtud ", " el philosopho mostro que las virtudes se causan en nos por las obras », "Aqui muestra que la virtud semejantes obras produze de aquellas de las quales se engendra $»^{30}$, révélant l'intérêt que cette idée d'acquisition de la vertu morale par l'habitude et les lois a suscité chez l'annotateur du texte, ou l'intérêt que selon lui le destinataire de la traduction y trouverait.

Un peu plus loin apparait en marge une définition simplifiée et condensée de la vertu morale. Le texte de la traduction propose une définition peu claire, « la virtud es habito elegible estante en medio quanto a nos es manifestado por el entendimiento segund que el sabio lo declarare $»^{31}$, dont les marges offrent une synthèse, « la virtud es abito electivo $»^{32}$.

Au livre III, à propos de la notion de délibération (bouleusis/consilium), Aristote indique ce qui en est la matière. Le texte de la traduction, une fois de plus, est assez confus :

Mas tomaremos consejo de las cosas que son en nos e son obrables. Ca estas son las que quedan que las causas paresçe que sean la naturaleza e la nesçessidad e la fortuna que sobre estas cosas es el entendimiento e toda cosa obrada que obra sea de onbre que todos los omnes toman consejo en las cosas que se obran por ellos [...] Quanto es la cosa mas dubdosa ha menester mas consejo [...] conviene tomar consejo en las cosas que vienen segund lo mas e non sabemos commo

\footnotetext{
${ }^{28}$ BNE, Ms. 10268, folio 11r.

${ }^{29}$ Ibid., folio $19 \mathrm{v}$.

${ }^{30}$ Ibid., folios $21 \mathrm{r}-\mathrm{v}$.

${ }^{31}$ Ibid., folio $27 \mathrm{r}$.

32 Ibid., folio 33r.
} 
vienen. E aun en las cosas que non son claras. Mas tomamos consejeros en las grandes cosas que non creemos seer bastantes nosotros para conosçer qual es mejor ${ }^{33}$.

Or une annotation marginale résume très clairement ce paragraphe, exprimant la notion de contingence dans une langue castillane courante et limpide: "Aqui muestra que conviene consejar de aquellas cosas en las quales no es determinado en que manera conviene fazer ».

Au livre VI, les développements consacrés aux diverses vertus intellectuelles sont très souvent traduits avec une grande maladresse, mais opportunément éclairés en marge par des annotations que j'indiquerai en italique. Ainsi, la notion de science :

Mas las cosas que acaesçen de seer en otra manera quando se fazen fuera de la vista son ocultas si son o si non son. Segund esto lo que es sabido por sçiençia nesçessario es. E luego es eterno, que todas las cosas que son por nesçessidad absoluta son eternas. Pues las eternas nin son generales nin corruptibles. Mas toda sçiençia paresçe seer demostrable [...] Por tanto el saber es habito demostrativo ${ }^{34}$. (en marge, «sçiençia es abito adquerido por silogismo demostrativo »)

Le paragraphe consacré à la notion de sagesse offre un très bon exemple de mauvaise traduction :

E por tanto es manifiesto que es mas verdadero (sic) en todos los saberes la sabiduria conprehendiente. E por esto conviene al sabio que non sepa las cosas sabidas de parte de los prinçipios solamente, salvo aun los prinçipios para dezir en ellos verdad. E por tanto sera la sabiduria entendimiento e saber [...] E ansi la sabiduria es una cosa çerca de todas las cosas e la prudençia es otra cosa çerca de las $\operatorname{cosas}^{35}$.

Cette dernière phrase, absolument incompréhensible, est à peine plus claire dans la version latine de Grosseteste, "sapiens idem omnes utique dicerent, prudens autem alterum ». En revanche, les annotations marginales en offrent une reformulation beaucoup plus claire:

Dize que el sapiente non solamente deve saber los prinçipios mas aun aquellas cosas que nasçen de los prinçipios e las conclusiones e saber dezir verdades çerca de los prinçipios, que en otra manera non son dichos sabios [...] Despues quel philosopho determino de cada una de las virtudes intellectuales, aqui muestra qual dellas sea mas noble e muestra que la sapiençia.

Même constat à propos des deux définitions de la vertu de prudence, l'une traduisant trop littéralement le latin de Grosseteste, «E pues ansi es, la prudençia non es saber nin arte [...] E ansi queda seer la prudençia abito verdadero hazedor con entender çerca de los bienes del omne e de los males », l'autre la résumant en la clarifiant en marge, « que la prudençia sea abito con razon çerca del bien e mal de omne $»^{37}$. marges :

Le lien entre prudence et vertu morale, de même, est reformulé de manière limpide dans les

E aun la obra cunple por la prudençia e virtud moral, mas la virtud moral faze la entinçion derecha. Mas la prudençia es la que aduze a esto [...] E si la entinçion es buena, sera laudable. Si es mala es astuçia. E por tanto los prudentes e los astutos se llaman agudos. Mas la prudençia non es esta potençia $[. .$.$] Nin sera el abito de la prudençia sin virtud moral [...] la maldad demuda e faze$ mentira çerca de los prinçipios de la obra. Por tanto es manifiesto que non puede seer el prudente si non bueno. ${ }^{38}$ (en marge, "Primero muestra que la prudençia non sea syn virtud moral», et plus bas

\footnotetext{
${ }^{33}$ Ibid., folios 39v-40r.

34 Ibid., folio 105 r.

35 Ibid., folio $108 \mathrm{r}$.

36 Aristoteles Latinus, op. cit., p. 260.

${ }^{37}$ Ibid., folios 106v-107r.

${ }^{38}$ Ibid., folios $115 \mathrm{v}-116 \mathrm{r}$.
} 
«Despues que el philosopho muestra que la prudençia non es sin virtud moral, aqui muestra que la virtud moral non es sin prudençia»)

Quand elles réalisent avec succès cette fonction d'éclairage et de synthèse, les annotations marginales du manuscrit BNE 10268 présentent le même avantage pédagogique que les gloses du prince Charles de Viana à sa traduction ou que la paraphrase abrégée de Nuño de Guzmán.

\section{Discordances et obscurités}

Toutefois, dans bien d'autres cas, loin d'éclairer le lecteur, les annotations marginales sèment la confusion en désignant certaines notions de l'Éthique par des termes différents de ceux choisis par le traducteur, sans qu'à aucun moment une incise dans la traduction ou en marge ne précise que ce sont deux termes désignant une seule et même notion.

Ainsi, au Livre III, la notion de choix (proairésis) est traduite par le mot « deliberaçion » dans le texte principal, «Ayuntemos con esto la deliberaçion que paresçe ser singular a la virtud e mas juzgar las condiçiones morales que por las obras »" ${ }^{39}$, et par « eleccion» dans les marges, « Despues que el philosopho determino de voluntario e involuntario, aqui determina de elecçion », et plus bas «aqui muestra que cosa es elecçion». En ceci, l'auteur des inscriptions marginales suit les choix de Grosseteste et de Bruni, tandis que celui du traducteur est bien malencontreux, puisque la délibération, opération qui précède le choix, est une autre notion de l'Éthique, certes généralement désignée par le terme de «consilium » (chez Grosseteste) ou «consultatio » (chez Bruni), mais aussi par le verbe « deliberare». Cette maladresse, qui pourrait ne pas être totalement gratuite si l'on considère l'importance de la notion de conseil aux yeux du Marquis de Santillane, sur laquelle je reviendrai, aboutit donc à mettre en avant à deux reprises l'idée de délibération. Elle sera d'ailleurs corrigée au Livre VI, où notre traducteur rejoint cette fois Grosseteste, «Por tanto pues que la virtud moral es abito elegible e la eleçion es apetito tomado por consejo, por esto conviene de seer la razon verdadera e el apetito recto si la election es buena ${ }^{40}$.

Au Livre VI, entre le texte central et les marges, trois termes différents désignent une même notion, celle d'intellect - « entender » et « entendimiento » dans le texte principal,

Las cosas en que sienpre dezimos verdad e nunca mentimos que pueden seer o non seer en otra forma son prudençia, sabiduria, entendimiento. E destas tres non es ninguno conviene a saber estas tres prudençia, sçiençia, sabiduria, pues queda que el entender es el que alcança los prinçipios, mais « intellecto » dans les marges, «Aqui determina de las virtudes intellectuales que son çerca dessos prinçipios, e primero de intellecto $»{ }^{41}$

Plus loin, à propos des vertus intellectuelles annexes à la prudence, que sont l'eubulia, vertu de celui qui délibère bien, la synesis, vertu de celui qui porte un jugement pertinent sur le fruit de la délibération, et la gnomè, vertu très proche de la synesis, mais qui porte sur l'équitable, de nouvelles discordances apparaissent entre la traduction et les inscriptions marginales.

Ainsi, la notion d'eubulia est traduite par « discreçion» dans le corps de la traduction, par trois orthographes de « eubulia » dans les notes marginales, et par « eubolia » entre les lignes de la traduction, de la main d'un correcteur qui a biffé « discreçion». De même, ce correcteur barre d'un trait les termes "cautela », remplacé par «eustochia», ainsi qu' «astuçia », remplacé par "solertia», substituant systématiquement aux termes castillans d'usage courant choisis par le traducteur les mots latins et translittérations depuis le grec de la version de Grosseteste. Il est

\footnotetext{
${ }^{39}$ Ibid., folio 37r.

${ }^{40}$ Ibid., folio $103 \mathrm{v}$.

${ }^{41}$ Ibid., folios $107 \mathrm{v}-108 \mathrm{r}$.
} 
malaisé de déterminer si annotations et corrections doivent être attribuées à la même personne, ou si le correcteur a modifié les choix du traducteur à la lumière des annotations marginales.

On trouve également deux orthographes de "synesis» dans les marges, "Despues que determino de enbulia aqui determina de sinisy e primero muestra que non sea sçiençia nin opinion » ou «Aqui muestra que senesis es abito de la sçiençia particular» tandis que le texte central traduit la notion par « cordura », et son contraire par « torpedad»,

Cordura e torpedad segund las quales dezimos cuerdos o torpes nin del todo es esso mismo que sçiençia o opinion [...] nin sera de las cosas perpetuas non movibles [...] mas de las cosas en que pone alguno dubda e se aconseja. E por tanto seran çerca de las cosas en que sera o de que sera la prudençia.. 42

Contrairement à l'auteur des notes marginales, qui reproduit simplement les translittérations de Robert Grosseteste, notre traducteur s'est efforcé de rendre les notions aristotéliciennes par des termes castillans compréhensibles, de même que Leonardo Bruni traduit «eubulia» par «bene consulere» et «bona consultatio», "synesis» et «asynesia» par «sagacitas » et «hebetudo », considérant que la langue latine est assez riche pour traduire ces concepts sans recourir aux translittérations barbares du « vetus interpres ».

Enfin, la notion de gnome est traduite par « aderesçamiento » dans le corps du texte, tandis que cette fois, ce sont les choix de l'auteur des annotations qui ne sont pas toujours cohérents : pour désigner les mêmes notions, tantôt il choisit les termes de Grosseteste, « Aqui muestra que sinesis no es generaçion de prudençia », "Aqui determina de la III virtud que se llama guome [sic] ", tantôt il reprend à son compte les choix du traducteur, "muestra que la cordura e el aderesçamiento son çerca de las cosas singulares $»^{43}$.

Notons que les notions qui ont suscité le plus de discordances entre le corps du texte et les annotations, révélant qu'il s'agissait de points ardus de la doctrine aristotélicienne, sont toutes liées à la prudence - choix et délibération, eubulia, synesis, gnome. J'ajouterai que dans l'un des très rares cas où les notes marginales ne sont pas plus claires que le texte de la traduction, la notion de prudence est également concernée : «Aunque la politica asi partia de ley commo la executiva sea prudencia, enpero manifiesto es visto es prudençia que es uno tan solo es çerca de si mismo $»^{44}$.

Tout ceci ne facilite guère la réception de la doctrine aristotélicienne de la prudence, alors même que certains indices semblent indiquer que cette notion suscitait un vif intérêt chez le destinataire et/ou le commanditaire de la traduction, intérêt qui aurait guidé le travail du traducteur, lui suggérant des choix tantôt fautifs, comme dans l'exemple déjà mentionné, « mas el que la prudençia ama pone su entendimiento en las cosas especulativas ${ }^{45}$, tantôt pertinents, comme dans l'exemple suivant, où notre traducteur a remplacé «bonum et sapiens» chez Grosseteste par « honesto e prudente », substituant la notion de prudence à celle de sagesse, car il est question d'affronter les aléas de la Fortune : «E pues el felix es honesto e prudente todos estimamos que devidamente conportara las ynfortunas e que de lo presençial sienpre fara obras nobles segund lo que se le ofresçiere $»^{46}$. Cette modification est bien conforme à la doctrine de l'Éthique, où la prudence est la vertu intellectuelle qui, appuyée sur l'expérience, guide les actes humains dans un monde contingent.

Or les citations du vade mecum des feuillets initiaux et finaux du manuscrit, nous le verrons, confirment l'importance du thème de la prudence aux yeux du possesseur du manuscrit, tandis que les autres vertus intellectuelles définies au Livre VI de l'Éthique ne semblent pas avoir autant retenu son attention. J'en proposerai plus loin une explication, à la lumière des conceptions politiques du Marquis et de son entourage. D'autres choix de traduction suggèrent que le

\footnotetext{
${ }^{42}$ Ibid., folio 113 r.

${ }^{43}$ Ibid., folio $113 \mathrm{v}$.

${ }^{44}$ Ibid., folio $110 \mathrm{r}$.

${ }^{45}$ Ibid., folio $196 \mathrm{v}$.

${ }^{46}$ Ibid., folio $13 \mathrm{v}$.
} 
traducteur a tenu compte du statut du destinataire de son travail ; ainsi, parmi les exemples de vertus morales proposés à la fin du Livre I, il a remplacé la notion de tempérance, sobrietas dans le texte de Grosseteste, par celle de justice, «la franqueza e justiçia, [son virtudes] morales » ${ }^{47}$, or la justice, vertu qui mieux que la tempérance sied au noble exerçant une juridiction sur ses terres, est un autre thème important des citations des feuillets de début et fin du manuscrit.

Que conclure de l'analyse de ces rapports entre le texte traduit et ses annotations marginales?

Peut-on supposer une double maladresse, d'une part le choix archaïque du texte latin de Robert Grosseteste au lieu de celui de Leonardo Bruni, d'autre part une incompréhension de l'auteur des notes marginales, qui, engoncé dans une culture toute scolastique encore, aurait corrigé la traduction conformément au texte de Grosseteste, sans comprendre que l'intention du traducteur, quoique maladroitement réalisée, était de transmettre l'Éthique d'Aristote dans une langue castillane courante et compréhensible de tous ?

Cette hypothèse est plausible, néanmoins j'aimerais en proposer une autre : une seule et même personne serait à l'origine de ces deux choix, celui de la version latine de base, et celui de la méthode de traduction - traduire tous les termes du texte de Grosseteste par des mots castillans courants. Ces choix seraient donc réfléchis et conformes à une conception cohérente de la manière dont la philosophie morale d'Aristote devait être transmise à un lectorat non universitaire et cependant exigeant : dans cette hypothèse, le traducteur aurait reçu la consigne de traduire l'Éthique conformément à ce qu'aurait été un consensus entre Alfonso de Cartagena et Leonardo Bruni, les deux adversaires de la fameuse controverse épistolaire à propos de la traduction latine de l'Éthique, autour de 1436. Comme je l'expliquais plus haut, dans le prologue de sa nouvelle traduction latine, Leonardo Bruni critique sévèrement la traduction de Robert Grosseteste, qualifié de "ferreum hominem», pour son manque d'élégance et son obscurité, notamment son choix de simplement translittérer les termes grecs de l'original - eutrapelia, bomolocbia, eustochia, etc-, et prône l'usage de termes latins courants et compréhensibles urbanitas, scurrilitas, rusticus, voluptas -, d'une langue naturelle et élégante, puisque la langue latine est assez riche pour traduire ces notions ${ }^{48}$. Dans son traité sur la traduction de l'Éthique, intitulé Declamationes super translatione Ethicorum Aristotelis, Alfonso de Cartagena répond aux arguments de Bruni et défend la version médiévale de Grosseteste, l'estimant plus rigoureuse et précise que celle de Bruni, et jugeant que la traduction d'un texte philosophique ne doit pas viser l'élégance mais avant tout la clarté :

Non ut Leonardum offendere, sed ut antiquum translatorem defendere temptem, ad conflictum accurro. Nec mihi curae est per cuncta libri Ethicorum vagari, sed veritatem translationis ex paucissimis demonstrare, ut illorum argumento cetera cognoscantur. ${ }^{49}$

Nec enim in philosophia verba sine freno laxanda sunt, cum ex improprietate verborum error ad ipsas res paulatim accrescat [...] Qui scientiarum districtissimas conclusiones eloquentiae regulis subdere vult, non sapit, cum verba addere ac detrahere ad persuasionis dulcedinem pertinet, quod scientiae rigor abhorret..$^{50}$

\footnotetext{
${ }^{47}$ Ibid., folio $18 \mathrm{v}$.

48 Humanismo y teoría de la traducción..., op. cit., p. 182-184.

${ }^{49}$ Humanismo y teoría de la traducción..., op. cit., p. 202. Traduction espagnole proposée dans cette édition : «me arrojo a la contienda no para intentar atacar a Leonardo, sino para defender al traductor antiguo. Y no es de mi interés dispersarme por la totalidad del libro de la Ética, sino demostrar a partir de unos quantos ejemplos la exactitud de la traducción ».

${ }^{50}$ Ibid., p. 214 et 232. «En filosofía no se pueden dejar libres y sin sujeción las palabras, porque un error debido a la falta de propriedad de éstas se acrecienta poco a poo hasta alcanzar las cosas mismas [...] el que quiere someter las conclusiones extremadamente rigurosas de las ciencias a las reglas de la elocuencia, no es juicioso, puesto que requiere añadir o suprimir palabras en aras del encanto de la persuasión, cosa que el rigor de la sciencia aborrece ».
} 
Plus généralement, c'est un débat sur la méthode de traduction qui oppose Robert Grosseteste et son défenseur castillan d'une part, et Leonardo Bruni d'autre part : tandis que la version de Grosseteste représente un exemple parfait de traduction mot à mot, "verbum de verbo », les humanistes optent pour la traduction "ad sententiam », telle que la défend Cicéron dans son De optimo genere oratorum, dont le but est de transmettre le sens tout en conservant les caractéristiques de la langue latine ${ }^{51}$.

Synthèse de ces deux approches, la traduction du manuscrit BNE 10268 comporte exclusivement des termes de la langue castillane courante, mais elle s'appuie sur le texte de Robert Grosseteste et non sur la nouvelle traduction latine de Leonardo Bruni, peut-être plus élégante, mais qu'Alfonso de Cartagena juge moins rigoureuse et précise dans le choix des termes que celle de Grosseteste.

Cette controverse était bien évidemment connue en Castille dans l'entourage de l'évêque de Burgos, tant à la cour de Jean II, à qui Bruni lui-même adressa plusieurs lettres, que dans les cercles du Marquis de Santillane, qui possédait dans sa bibliothèque la copie de ces lettres de Bruni et de son traité sur la chevalerie ${ }^{52}$ et la traduction castillane faite par son écuyer Martín de Ávila d'un traité de Pier Candido Decembrio ${ }^{53}$, compagnon de Bruni et adversaire de Cartagena dans la controverse sur la traduction de l'Éthique; en outre, dans une lettre à son fils Pedro González de Mendoza, futur Gran Cardenal d'Espagne, alors étudiant à Salamanque, le Marquis évoque l'un de ses amis, probablement Nuño de Guzmán, récemment revenu d'Italie avec des traductions de Leonardo Bruni ${ }^{54}$.

Plusieurs figures pourraient être à l'origine de cette consigne donnée au traducteur: Alfonso de Cartagena lui-même, dont on sait qu'il eut d'autres occasions de s'entretenir des écrits de Leonardo Bruni avec son ami le Marquis ${ }^{55}$, quelque autre érudit de l'entourage du Marquis ayant pris connaissance des termes du débat entre Bruni et Cartagena, comme Pedro Díaz de Toledo, chapelain du Marquis, ou encore le fils du Marquis, Pedro González de Mendoza, qui étudia les textes d'Aristote à l'Université de Salamanque entre 1446 et 1452, à savoir précisément avant l'adoption du texte de Bruni comme texte de référence par Pedro Martínez de Osma, nouveau professeur de philosophie morale à partir de $1457^{56}$.

Quant aux annotations, elles ont pu être ajoutées dans un second temps à un texte originellement conçu pour fonctionner seul, mais elles peuvent fort bien, et cette hypothèse me convainc et me séduit davantage, avoir été d'emblée incluses dans ce projet pédagogique initial, clarifiant et résumant d'une part certains passages pour faciliter la lecture, indiquant d'autre part en marge les termes désignant les notions clés dans la version de Grosseteste, pour des raisons à la fois de simple érudition, d'utilité - donner accès à d'autres textes de vulgarisation de l'Éthique qui utilisent également ces termes, incluant ainsi le lecteur dans une communauté éclairée

\footnotetext{
51 Ibid., introduction, p. 72 et 76.

52 BNE, Ms. 10212, contient aussi la traduction castillane d'un résumé de l'Éthique par Bruni, l'Isagogicon moralis disciplinae.

53 SCHIFF, Mario, La bibliothèque..., op. cit., p. XC.

${ }^{54}$ Ibid., p. 452.

$55 \mathrm{Il}$ répondit ainsi à la Qüestión du Marquis sur la chevalerie, suscitée par la lecture du traité de Bruni sur ce thème.

${ }^{56}$ Rien ne semble prouver, en effet, que le texte de l'Éthique utilisé comme support de cours à la Faculté des Arts de l'Université de Salamanque était, avant que Pedro Martínez de Osma n'accède à la chaire de philosophie morale en 1457, la version de Bruni. Les fragments des statuts du Pape Martin V octroyés à l'Université en 1422, partiellement édités par Enrique Esperabé Arteaga dans Historia pragmática e interna de la universidad de Salamanca, Salamanca, Francisco Núñez Izquierdo, 1914, ne font allusion à l'Éthique qu'à travers l'expression «philosophia moralis». Ce volume ne donnant qu'un «sucinto extracto» des Statuts, j’ai également consulté un incunable de 1487 les reproduisant intégralement, où je n'ai trouvé aucune mention de la version de l'Éthique à étudier, mais seulement de la «philosophia moralis» à l'article 16, comme matière obligatoire à étudier à la Faculté des Arts, et à l'article 19, à propos du titre de Bachelier in Artibus. Aucune mention explicite de l'Ethique ni d'Aristote n'apparait. Il semble même plus probable que la version de l'Éthique utilisée par le prédécesseur de Pedro Martínez de Osma, fray Martín de Córdoba, ait été celle de Grosseteste et non celle de Bruni, comme tend à le suggérer la comparaison entre les fragments de son Compendio de la Fortuna où il paraphrase l'Éthique, et les versions latines de Grosseteste et Bruni.
} 
partageant certains concepts et les termes techniques qui les désignent, eutrapelia, gnome, synesis etc -, et d'efficacité pédagogique - si la traduction castillane était pensée pour accompagner la lecture du texte latin.

Seulement, les personnes chargées de mettre en œuvre cette conception, à la fois le traducteur et l'annotateur, se seraient acquittées de cette tâche avec maladresse ${ }^{57}$, omettant de préciser, notamment, que plusieurs termes renvoient à une unique notion, par exemple synesis et cordura. Quant au correcteur, il aurait ajouté une maladresse supplémentaire en s'appuyant sur les annotations marginales pour corriger le texte, sans comprendre que les deux étaient pensés pour se compléter.

Qui étaient le traducteur et l'annotateur? Il semble peu probable que le traducteur soit Pedro Díaz de Toledo, chapelain du Marquis auteur de plusieurs traductions, car sa prose, telle qu'elle apparaît dans le Razonamiento en la muerte del Marqués de Santillana ${ }^{58}$, est bien plus élégante que notre laborieuse traduction verbum de verbo. Quant aux annotations, il est certes tentant de les attribuer à un illustre membre de l'entourage du Marquis, comme Cartagena lui-même, Pedro Díaz de Toledo ou le futur grand Cardinal, mais les discordances signalées plus haut suggèrent d'autres candidats moins illustres, parmi les letrados au service du Marquis, tels «Fernandus Guterii de Cardoso in artibus bachalarium », copiste du manuscrit BNE 10269 contenant des textes de vulgarisation de l'Éthique, ou Alfonso de Zamora, «bachiller en decretos ». Parmi ces serviteurs, formés à la Faculté des Arts ou en Droit, certains se virent confiés des travaux de copie ou de traduction ${ }^{59}$.

Il est également possible que le premier destinataire de cette traduction ait été le roi Jean II lui-même : dans ce cas, le Marquis aurait fait recopier par Morillo un manuscrit de la bibliothèque royale, comme il le fit pour la traduction de l'Isagogicon moralis disciplinae de Leonardo Bruni, conservée dans le manuscrit BNE 10212 ${ }^{60}$. Le traducteur serait dans ce cas un serviteur du roi, de profil semblable à celui des bachilleres du Marquis; Alfonso de Cartagena, membre du Consejo Real, comme Pedro Díaz de Toledo, qui fut au service du roi dans les années $1440^{61}$, pourraient être à l'origine du projet, comme dans la première hypothèse.

Quoi qu'il en soit, cette traduction est à l'image de la bibliothèque du marquis de Santillane, ouverte aux classiques de l'Antiquité et à l'humanisme italien, mais comptant aussi nombre d'œuvres proprement médiévales. Surtout, même dans l'hypothèse où elle aurait initialement été réalisée pour le roi, les thèses contenues dans l'Éthique correspondent davantage aux inquiétudes politiques de l'entourage du Marquis, pour qui leur traduction à la fois claire et précise présentait une utilité, notamment la traduction de la doctrine de la prudence : celle-ci séduisait certes les humanistes italiens pour la valorisation de l'agir politique ici-bas qu'elle autorisait, pour le bonheur proprement politique qu'elle attribuait aux recteurs de la Cité (comme Leonardo Bruni

\footnotetext{
${ }^{57}$ Ainsi, la décision de traduire toutes les notions de l'Éthique par des termes de la langue castillane courante a été appliquée de manière systématique et parfois fort incongrue : les trois formes correctes de régimes politiques, monarchie, aristocratie, démocratie (ou régime constitutionnel), dont Alfonso de Cartagena dit dans sa défense de Grosseteste «Pero ya el hecho de que haya dejado sin traducir vocablos griegos es una acusación que debe parecer sorprendente a los hombres instruidos, puesto que nos servimos de no pocas palabras griegas ", cf. Humanismo y teoría de la traducción..., op. cit., p. 209, sont ici désignées par des termes trop vagues, reyno, señorío, abilidad.

${ }^{58}$ Édité par PAZ Y MELIA A., Opúsculos literarios, Madrid, Sociedad de Bibliófilos Españoles, 1892, p. $247-356$.

${ }^{59}$ Le Marquis confia à Alfonso de Zamora la traduction castillane d'un Paul Orose aragonais, et à son écuyer Martín de Ávila celle d'un traité de Pier Candido Decembrio. Cf SCHIFF, Mario, La bibliothèque..., op. cit., p. LXXXIV et p. 423.

${ }^{60}$ JIMÉNEZ SAN CRISTÓBAL Montserrat, El Isagogicon moralis disciplinae de Leonardo Bruni y sus versiones castellanas, thèse de doctorat soutenue en 2010, p. 410.

${ }^{61}$ Dans un tableau sur les commanditaires et destinataires des traductions du XVe siècle, Carlos Alvar mentionne les traductions de l'Ethique par Pedro Díaz de Toledo en 1443-44 pour Juan II, sans autres précisions. Cf Traducciones y traductores..., op. cit., p. 286. La seule traduction liée à l'Éthique que revendique l'intéressé est celle de la Summa Alexandrinorum, un résumé de l'Éthique, cf Repertorio de traductores del siglo XV, op. cit., p. 76. Mais c'est une traduction du texte intégral que contient le manuscrit BNE 10268.
} 
lui-même, chancelier de Florence), mais elle semble avoir suscité un intérêt non moins vif, quoique pour d'autres raisons, de la part du Marquis et de son entourage.

\section{Aux marges du manuscrit : un vade mecum pour le Marquis de Santillane?}

Dans les pages qui suivent, j’analyserai les citations extraites d'œuvres aristotéliciennes, éparses sur les feuillets initiaux et finaux du manuscrit ${ }^{62}$, écrites de plusieurs mains. Ces autres marges, qui précèdent et suivent la traduction de l'Éthique, ne sont pas sans lien avec celles du texte traduit, commentées ci-dessus, puisque certaines de ces mains ont également annoté la traduction.

Toutefois, les citations des premiers et derniers feuillets, probablement copiées par des lecteurs de la traduction castillane, ne sont pas extraites de celle-ci : elles juxtaposent le texte latin de Robert Grosseteste et une traduction castillane qui n'est pas celle du manuscrit, mais qui a dû être improvisée par la personne ayant copié les citations latines.

Mises à part quelques citations de saint Thomas et de saint Augustin, celles-ci proviennent dans leur grande majorité d'œuvres aristotéliciennes, et sont liées à des thèmes éthiques qui pouvaient intéresser le Marquis ou les membres de son entourage, comme les définitions aristotéliciennes de l'honneur, de la vertu, du courage. Entre deux séries de citations apparait également une présentation de l'illustre ascendance de doña Leonor de La Vega, mère du Marquis, dont les ancêtres seraient des frères du roi de France : " dos hermanos del rey de Francia $[\ldots]$ vinieronse a Castilla $»^{63}$.

Dans ces citations, les thèmes politiques revêtent une importance particulière. L'intérêt des lecteurs et possesseurs de ce manuscrit pour ces thèmes peut s'expliquer à la lumière du contexte politique, le règne de Juan II et/ou celui de son successeur Enrique IV, alors que les livres du Marquis étaient échus en héritage à son héritier, don Diego Hurtado de Mendoza : en effet, les citations sont de plusieurs mains et peuvent avoir été copiées à des dates différentes.

Parmi ces thèmes politiques, apparaît un résumé de l'opposition entre formes politiques correctes et corrompues qu'expose Aristote dans la Politique, ainsi que, de manière condensée, dans l'Éthique: "Tres policias ay buenas e tres malas. Las buenas son rreyno e aristocracia e policia $»^{64}$.

Plusieurs citations portent sur le thème de la justice, qui pouvait intéresser les grands nobles castillans exerçant une juridiction sur leurs terres : deux définitions de la justice tirées du livre $\mathrm{V}$ de l'Éthique apparaissent au folio 1 verso. Souvenons-nous également de la modification introduite par le traducteur dans le texte de l'Éthique, remplaçant la notion de tempérance par celle de justice parmi les exemples de vertus morales.

Un autre thème récurrent est la royauté : une citation oppose le roi véritable et le roi «según opinión », « dize Aristotiles que ay rey segund opinion e rey segund verdad » donne la définition du roi selon le livre VIII de l'Éthique, «Non enim est rex que non est per se suficiens et omnibus bonis super excelens. Tallis [sic] autem nullo indiget. Non ciertamente es rey el que no es suficiente por sy y en todos los bienes mas excelente $»^{66}$.

\footnotetext{
${ }^{62}$ Ces citations éparses apparaissent sur les quelques feuillets qui précèdent la traduction castillane de l'Éthique, puis sur les quelques feuillets qui la suivent. En outre, ces pages ont reçu une foliotation moderne qui n'est pas celle de la traduction, foliotation qui va de 1 à 5 au début puis à nouveau de 1 à 5 à la fin du manuscrit, c'est pourquoi je préciserai dans chaque citation, en plus du numéro du feuillet, s’il se situe dans les feuillets du début ou de la fin du manuscrit.

${ }^{63}$ Feuillets initiaux, folio $4 \mathrm{r}$.

${ }^{64}$ Feuillets initiaux, folio $2 \mathrm{r}$.

${ }^{65}$ Feuillets initiaux, folio $1 \mathrm{r}$.

${ }^{66}$ Feuillets initiaux, folio $1 \mathrm{v}$.
} 
Le thème du conseil, devoir du vassal, apparait également : « Non consiliamur de fine set de his que sunt ad finem : non consejamos de fin mas de las cosas que son para este fyn ${ }^{67}$. En réalité, il s'agit de la notion de délibération (bouleusis), traduite en latin par "consilium » dans la traduction de Robert Grosseteste.

Parmi ces divers thèmes politiques, certains étaient susceptibles d'intéresser le Marquis de Santillane dans le contexte de l'opposition entre la haute noblesse et Álvaro de Luna, privado du roi, l'attitude du Marquis ayant été fort ambiguë dans ce conflit: je songe à l'opposition entre «alevoso » et «traydor» au folio $5 \mathrm{r}$ au début du manuscrit, ou à la définition des passions, «Passibilidades e afeçiones » au folio $5 \mathrm{v}$ à la fin, parmi lesquelles sont mentionnées la haine et la colère qui guident le tyran dans ses décisions, thème dont les troubles du règne suivant, celui d'Enrique IV, peuvent également éclairer la récurrence dans ces feuillets.

J'aimerais surtout souligner l'importance du thème de la prudence dans cet ensemble de citations qui forment une manière de vade mecum. La prudence y apparait d'une part comme vertu morale, dans sa définition tirée du livre VI de l'Éthique: "La prudencia es habito verdadero hazedor con razon çerca de los bienes de los onbres e desechador de los males ${ }^{68}$. Notons que contrairement aux citations précédentes, qui juxtaposaient le texte latin et sa traduction castillane, cette définition apparaît directement en castillan. Le mot « entender » est barré est remplacé par « razón»; l'adjectif «bueno» est ajouté entre les lignes pour qualifier cet habitus. Le copiste explicite par cet ajout le rapport étroit entre la prudence, vertu intellectuelle, et les vertus morales, dont elle détermine le juste milieu. Souvenons-nous que les annotations marginales qui accompagnent la traduction castillane de ce même manuscrit soulignent également ce lien entre prudence et vertu morale : «Primero muestra que la prudençia non sea syn virtud moral », et plus bas «Despues que el philosopho muestra que la prudençia non es sin virtud moral, aqui muestra que la virtud moral non es sin prudençia »"

Cependant, c'est surtout comme vertu politique que la prudence fait l'objet de plusieurs citations.

Dès les premiers feuillets, apparaît l’idée que la prudence est la vertu propre du monarque,

Prudencia autem principis propia virtus sola, allias enim virtutes videbitur esse nescesarium comunes subditorum et principum. Subditi autem non est virtus prudencia set opinio vera. La verdadera virtud del principe es la prudencia ${ }^{70}$,

puis de nouveau dans les feuillets finaux, «aun que le tengamos por rey sy no es prudente no sera rey verdadero segund opinion » référence.

Simultanément, dans ces mêmes feuillets finaux, la prudence apparaît comme la vertu du conseiller: «La prudençia deve tener tres virtudes consigo. La primera es ebulia [...] La ebulia es rectitud del consejo [...] Ebulia es tanbien rectitudo consilii ad finem bonum semper per vias congruas $\gg{ }^{71}$.

Mais allons plus loin. À première vue, ces citations sont éparses, simplement juxtaposées sans suivre nul ordre logique ni thématique; toutefois, considérées dans leur ensemble, elles s'emboîtent comme les pièces d'un raisonnement unique, se complètent en composant un syllogisme multiple, où s'esquisse la figure d'un roi prudent, dont les actes de gouvernement seraient le résultat d'une délibération collective, menée conjointement avec les grands nobles du royaume, la prudence étant, dans cette configuration, la vertu commune au roi conseillé et à ses conseillers nobles.

En effet, la citation des premiers feuillets, « dize Aristotiles que ay rey segund opinion e rey segund verdad», est complétée dans les feuillets finaux par une autre, qui révèle que le critère

\footnotetext{
${ }^{67}$ Feuillets initiaux, folio $1 \mathrm{v}$.

${ }^{68}$ Feuillets initiaux, folio $1 \mathrm{v}$.

${ }^{69}$ Folio 116r-v de la traduction castillane de l'Éthique.

${ }^{70}$ Folio 1v.

${ }^{71}$ Folio 5 r.
} 
distinguant le vrai roi et le roi « según opinión » n’est autre que la vertu de prudence, «Rey es el que tiene naturalmente inclinaçiones de virtud e [...] tenga la virtud moral que es la prudencia $[\ldots]$ aun que le tengamos por rey sy no es prudente no sera rey verdadero segund opinion ».

Si ces deux citations sont comparées à l'énumération des formes correctes et corrompues de régimes politiques, les similitudes de formulation suggèrent que c'est la vertu de prudence qui distingue le roi du tyran :

Tres policias ay buenas e tres malas. Las buenas son rreyno e aristocracia e policia [...] Las tres policias malas e contrarias destas son la primera tyrania [...] este tal no es rrey, aunque le tengan por rrey, sy no tyrano pues que no mira el bien de rreyno sy no su bien particular. ${ }^{72}$

De même, le thème du conseil évoqué dans les premiers feuillets, «Non consiliamur de fine set de his que sunt ad finem ; non consejamos de fin mas de las cosas que son para este fyn », est ensuite, dans les derniers feuillets, mis en rapport avec la prudence, à travers la notion d'eubulia, vertu annexe de la prudence, «La prudençia deve tener tres virtudes consigo. La primera es ebulia [...] La ebulia es rectitud del consejo ». Ainsi, la prudence apparaît comme la vertu de celui qui conseille, le conseil étant l'un des devoirs du bon vassal, mais elle est également attribuée au roi comme vertu propre; cet ensemble de citations dispersées propose donc la prudence comme vertu commune au souverain qui délibère et se laisse conseiller, et à ses sujets.

Un raisonnement à l'intérieur du raisonnement vient confirmer cette thèse, en faisant appel à une notion issue des textes de saint Jérôme et saint Ambroise, ensuite adoptée par les scolastiques, la comparticipatio. La première partie du raisonnement consiste à attribuer à tous ceux qui partagent les vertus propres au roi - à savoir la vertu morale et la prudence, toutes deux étant indissolublement liées - une participation à l'essence royale :

El que toviere inclinaçiones buenas naturalmente a virtud e tuviere la virtud moral, este sera conpartiçipiante al que llamamos rey porque es prudente [...] Qualquiera que fuera inclinado bien a misura e fuere prudente sera conparticipante al rey verdadero. ${ }^{73}$

Je me permets ici de rappeler l'ajout de l'adjectif «bueno » pour caractériser l'babitus de prudence, dans la définition de la prudence au folio $1 \mathrm{v}$.

Or - et c'est la deuxième partie du raisonnement - telle est la définition du noble telle qu'elle apparait sur le même feuillet en fin de manuscrit, accompagnée des définitions de l'homme libre et de l'bidalgo: "Libre es el que tiene inclinaçiones a virtud [...] Noble es el que tenga las inclinaçiones que estos $\left[=\mathrm{el}\right.$ libre y el hidalgo] tienen $»^{74}$. Cependant, un autre critère définissant la noblesse est l'ancienneté du lignage, de son pouvoir et de sa richesse : « Noble es el que tenga las inclinaçiones que estos tienen [...] e que venga ya muy lexos [...] a lo menos de bisabuelo, e que ayan seydo ricos e poderosos estos de los que viene el noble ». Souvenons-nous, à ce propos, du paragraphe sur les frères du roi de France, ancêtres de la mère du Marquis, parmi les citations des feuillets initiaux.

Ainsi, considérées comme les pièces d'un unique système argumentatif, comme autant de propositions mineures et majeures disjointes, dont les conclusions demeurent implicites, les citations et fragments dispersés sur les premiers et derniers feuillets du manuscrit BNE 10268 suggèrent la conception néo-féodale d'un roi "primus inter pares ", caractérisé par un ensemble de vertus qu'il partage avec les grands nobles du royaume.

\footnotetext{
${ }^{72}$ Cette paraphrase castillane de l'Éthique n'a aucun rapport avec la traduction de ce même passage dans ce même manuscrit : «De la poliçia son tres especias [...] son de la poliçia reynado e poderio e abilidad. El reyno es que todos obedescan a un (sic) que se enseñoree dellos. E señorio es que sean muchos e buenos enseñoreados. E abilidad es que se enseñoreen los onbres del comun », folio 258v. Comme dans les autres cas commentés plus haut, les mots grecs ont été éliminés.

${ }^{73}$ Folio $4 \mathrm{v}$, feuillets finaux.

${ }^{74}$ Feuillets initiaux, $4 \mathrm{v}$.
} 
La conception du pouvoir royal comme poderío absoluto, défendue à la même époque par Álvaro de Luna, privado du roi et grand ennemi du Marquis, telle qu'elle est formulée dans les ordonnances des Cortes d'Olmedo (1445), était tout autre :

Que ninguno non sea osado de tocar en su rrey e prínçipe commo aquel que es ungido de Dios nin aun de rretraer nin dezir del ningunt mal nin aun lo pensar en su espíritu, mas que aquel sea tenido commo vicario de Dios e onrrado commo por esçelente [...] el rey cuyo coraçon es en las manos de Dios, e lo el guia inclina a todo lo quel plaze, el qual es vicario e tiene su logar en la tierra $[\ldots]$ el su poderio non lo ha de los omes mas de Dios, cuyo logar tiene en todas las cosas temporales. ${ }^{75}$

On retrouve ici l'image d'un roi incarnant la scientia ou la sapientia - les ordonnances du début de la période Trastamare recourent de plus en plus fréquemment à l'expression « ex mea certa scientia $»^{76}-$, vertus intellectuelles seyant mieux au monarque en tant que vicaire de Dieu sur terre, plus nobles que la modeste prudence aristotélicienne, faillible, attachée à un ici-bas soumis à la contingence et excluant toute possibilité de certitude.

Bien qu'étant écrites de plusieurs mains et peut-être à des dates différentes, ces citations composent ensemble, par la sélection qu'elles suggèrent, un unique raisonnement, où chacune complète les autres. Elles peuvent avoir été couchées sur ces feuillets par les copistes au service du Marquis : on trouve en effet dans d'autres manuscrits ayant appartenu à la bibliothèque de Guadalajara des citations inscrites dans les marges par ces serviteurs polyvalents, parfois également traducteurs, comme Alfonso de Zamora, copiste du manuscrit BNE 10144 où il écrivit «Lictera Bononiensis: Omnium habere memoriam et in nullo penitus errare pocius divinitatis quam humanitatis est. Zamorensis Alfonso Bachalarius $»^{77}$. Il est possible que ces citations proviennent de notes de cours et textes utilisés en classe qu'aurait rapportés à Guadalajara le fils du Marquis, Pedro González de Mendoza, étudiant à Salamanque de 1446 à 1452 ; elles y auraient été copiées par les bachilleres au service de son père, qui auraient sélectionné et traduit les citations en castillan - le Marquis déplorait de ne pas savoir le latin ${ }^{78}$.

En effet, les citations de ces feuillets initiaux et finaux semblent provenir de cours de philosophie morale, portant principalement sur l'Éthique, tels qu'ils étaient dispensés dans les studia generalia comme celui de Salamanque, au sein de la Faculté des Arts ${ }^{79}$. À l'époque où Pedro González de Mendoza y étudiait, la chaire de philosophie morale était occupée par fray Alonso Martín de Córdoba, dont le contenu des cours n'est pas connu. En revanche, celles de son successeur, Pedro Martínez de Osma, magister de philosophie morale à partir de 1457, ont été conservées. Or les thèmes abordés dans les citations du manuscrit 10268 correspondent aux grandes questions commentées dans son cours, comme la définition de la noblesse. Voici celle de Pedro Martínez de Osma dans son commentaire de la Politique d'Aristote : "nobiles dicuntur illi solum qui talem inclinationem ex parentibus traxerunt primis, secundis, tertiis et quartis, et ita, secundum quamdam antiquitatem $»^{80}$.

Quant à la prudence qui nous occupe, Pedro de Osma affirme, conformément aux thèses suggérées par les citations de notre manuscrit, que les connaissances utiles au roi comme

\footnotetext{
75 Cité par NIETO SORIA, José Manuel, «El poderío real absoluto de Olmedo (1445) a Ocaña (1469): la monarquía como conflicto », in En la España medieval, 21, 1998, p. 159-228.

${ }^{76}$ Ibid.

${ }^{77}$ Folio 227 v.

${ }^{78}$ SCHIFF, Mario, La bibliothèque ..., op. cit., p. LXIII.

79 Outre les citations analysées ci-dessus, y figurent aussi plusieurs présentations schématiques à valeur mnémotechnique, binaires, ternaires ou quaternaires, recourant à un vocabulaire scolastique : "Quatro grados ay de anima, el primero vegetatyvo, e el segundo sensytivo secundum locum motivus [...]», "Duplex est noticia speculativa, scilicet propter quid et quia », "Artes liberales sunt septem », "Virtutes theologales sunt tres [...] Virtudes cardinales sunt quatuor».

${ }^{80}$ MARTÍNEZ DE OSMA, Pedro, Comentario a la Política de Aristóteles, LABAJOS ALONSO José (éd.), Salamanca, Publicaciones Universidad Pontifica, 2006, p. 474.
} 
législateur ne sont pas les mathématiques ou l'astronomie mais, précisément, la matière de la prudence :

Quae sint necessaria principem scire; quae si ignoret, non solum ignorantia non excusabit eum, verum ignorantia talis erit peccatum in ipso. Incumbit enim principi [...] quae necessaria sunt ad regimen civitatis scire; unde patet, quod non est necessarium quod princeps sit astrologus, ut dominus Illefonsus, rex Castellae, nec phisicus nec dialecticus. Et praeterea, recte dicebat Euripides : non mihi speciosa quaero; vel, ut altera translatio dicit, quae vana et alta. Qualia sunt quae philosophi considerant, sed debet ea adiscere et scire unde legislator fieri possit; unde debet habere prudentiam architectonicam, id est, praesidentem, quae alias legis positiva nominatur; et ita debet ea legere, unde hujusmodi prudentia adquirere possit. ${ }^{81}$

La doctrine exposée ici par Pedro de Osma démontre une parfaite fidélité à la pensée d'Aristote, selon qui la loi est dictée par la prudence et l'entendement, et non par la sagesse. Pedro de Osma rejette ici explicitement le modèle alphonsin du rex sapiens, dont l'action politique se fonderait sur des connaissances de nature scientifique, et manifeste une conception plus immanente et horizontale de l'agir politique, caractéristique de l'humanisme politique. Notons que la citation du livre $\mathrm{X}$ de l'Éthique à propos de la loi apparaît justement parmi les citations du dernier feuillet du manuscrit 10268, "Lex est sermones ab aliqua prudentia et intellectu procedentes. La ley es palabras que proceden de alguna prudencia e entendimiento ${ }^{82}$.

Ainsi s'épanouit, dans les cercles de la haute noblesse castillane et des studia generalia des règnes de Juan II et d'Enrique IV, un âge d'or de la prudence aristotélicienne, qui se prolongea au temps du fils aîné du Marquis, Diego Hurtado ${ }^{83}$, et jusqu'au second duc de l'Infantado, qui réunit lui-même une cour littéraire à La Alcarria, et privilégia, comme son illustre ancêtre, les écrits d'Aristote en matière de philosophie morale ${ }^{84}$. Dans le Tractado de la bienaventurança qu'il lui dédie, le docteur Fernán Núñez, membre du Consejo Real des Rois Catholiques, rappelle que le duc affirmait volontiers qu'Aristote était celui qui avait le mieux traité le thème du bonheur, ce pourquoi il se propose, par ce traité, de «acallar la apología aristotélica » ${ }^{85}$. Ainsi s'annonçait le déclin progressif de l'influence de l'Éthique d'Aristote sur la pensée politique des élites castillanes ; dans un même mouvement, les Rois Catholiques remettaient la haute noblesse à sa place, tandis

${ }^{81}$ Ibid., p. 394.

${ }^{82}$ En revanche, dans la traduction latine (1240) par Hermann l'Allemand du commentaire de l'Éthique par Averroès, intitulé Liber Nicomachie - dont on retrouve des passages dans les Siete Partidas -, le terme «prudentia » est remplacé par celui de «scientia » : "lex est sermo procedens a scientia et intellectu », manuscrit 94-14 Bibliothèque Capitulaire de la cathédrale de Tolède, folio 73r. Implicitement, cela suggère que la loi procède de la sagesse, sapientia, qu'Aristote définissait comme la réunion de la science et de l'intellect. Une telle conception trahissait certes la pensée d'Aristote, mais elle entrait en parfaite consonance avec la conception alphonsine de la royauté. L'original arabe du commentaire d'Averroès étant perdu, il est difficile de déterminer si cette modification est d'Averroès lui-même ou du traducteur Hermann, lui-même influencé par une conception de la royauté que l'entourage de Ferdinand III avait commencé à ébaucher, et qui se préciserait sous Alphonse X. La prudence d'Aristote, clairement distinguée de la science et de la sagesse, pouvait apparaître trop modeste pour un roi vicaire de Dieu sur terre : «prudentia non est scientia, neque etiam est magisterium [...] prudentia universaliter est habitus animalis efficiens cum ratione veridica bonorum humanorum [...] Et ex quo est iste habitus partis animae quae habet rationem, intendo partem rationalem, tunc ergo iste habitus, intendo prudentialem, est virtus illius partis quae habet putationem. Putatio enim est in rebus contingentibus », Liber Nicomachie, Bibliothèque Capitulaire de la cathédrale de Tolède, Ms. 94.14, folio 38v.

${ }^{83}$ Le premier duc de l'Infantado accorda beaucoup d'importance à la bibliothèque de son père, au point d'en faire une partie du mayorazgo, comme il l'explique dans son Testament, reconnaissant la valeur et l'utilité proprement politiques des écrits conservés dans la bibliothèque : «mando al Conde mi fijo [...] los libros que en mi librería y cámera se fallaren [...] esto porque yo deseo mucho que él e sus descendentes se den al estudio de las letras commo el Marqués mi señor [...] creyendo mucho por ello ser crecidas e alçadas nuestras personas e casas ». Publié par LAYNA SERRANO, Francisco, Historia de Guadalajara y sus Mendoza, Madrid, Aldus, 1942, p. 474.

${ }^{84}$ Alfonso de Cartagena, ami du Marquis, avait souligné dans plusieurs de ses écrits la supériorité de la philosophie morale d'Aristote par rapport à celle des stö̈ciens.

${ }^{85}$ L’expression est de Carmen Parrilla. Voici les termes de Fernán Núñez : «Vuestra señoría afirmativamente quiso sostener que el mismo Aristótiles escrivió e conosció mejor que otro mundano omne de la bienaventurança ». Voir l'édition du Tractado par PARRILLA, Carmen, in Lemir, 15, 2011, p. 1-67, p. 2-3. 
que les auteurs de traités politiques de leur entourage remettaient à la sienne, celle de vertu intellectuelle subalterne, l'embarrassante prudence aristotélicienne.

\section{Conclusion}

L'analyse de ces deux types de marges dans le manuscrit BNE 10268 révèle l'intérêt suscité par la notion aristotélicienne de prudence chez les lecteurs de l'illustre bibliothèque de Guadalajara, dans deux perspectives bien différentes néanmoins. Les annotations marginales qui accompagnent la traduction castillane de l'Éthique cherchent à faciliter la lecture et la compréhension du texte, ceci en le clarifiant par la paraphrase, ou en le complétant, notamment par l'ajout, à côté des termes castillans courants utilisés dans la traduction, les termes grecs translittérés désignant les mêmes notions, ceci dans une visée de simple érudition d'une part, d'utilité morale d'autre part, les lectures de philosophie morale étant considérées comme utiles pour pratiquer la vertu ${ }^{86}$.

Les citations qui couvrent les feuillets finaux et initiaux du manuscrit, en revanche, révèlent qu'une sélection de thèmes a été opérée au fil de la lecture de l'Éthique, laquelle semble avoir été guidée non pas par un intérêt purement moral ou d'érudition, mais aussi et surtout par un intérêt politique. Au-delà de l'attention que le Marquis, ses descendants et ses serviteurs pouvaient porter à l'Éthique d'Aristote en tant que maîtresse de vertu, elle était également pour eux l'objet d'un intérêt proprement politique, lequel se portait tout particulièrement sur la notion de prudence.

Les autres textes de vulgarisation de l'Éthique que possédait le Marquis élèvent également la prudence au rang de principale vertu politique, vertu du citoyen dans le Libro della vita civile de Matteo Palmieri, du législateur et du gouvernant dans l'Isagogicon moralis disciplinae de Leonardo Bruni, des nobles «rectores» des environs de Rimini dans le Liber de virtutibus de Guido Vernani, «prudentia inter alias magis convenit rectoribus et statui militari $»^{87}$.

En revanche, la doctrine des vertus intellectuelles exposée dans l'Éthique pouvait être dérangeante du point de vue du monarque. Il me semble, et c'est ce que je tâche de démontrer dans ma thèse de doctorat, que la prudence d'Aristote, vertu propre du législateur et du gouvernant dans l'Éthique et la Politique, est restée une vertu royale problématique dans les écrits émanant de la royauté, les rois revendiquant bien plutôt la vertu de sapientia, plus parfaite, non faillible, détachée du socle du sensible et de l'expérience sur lequel s'appuie la prudence. Ainsi, il est révélateur que dans les Siete Partidas d'Alfonso X el Sabio, l'Éthique soit citée à propos de certains thèmes, y compris politiques comme la différence entre le roi et le tyran, mais pas à propos de la prudence, alors même que le schéma des vertus cardinales, où figure cette dernière, est utilisé pour énumérer les vertus du roi.

\footnotetext{
${ }^{86}$ Cet intérêt pour l'utilité morale des textes de vulgarisation de l'Éthique d'Aristote est manifeste dans le manuscrit BNE Res/244, ayant également appartenu au Marquis, et contenant une copie du Libro della vita civile de Matteo Palmieri. Au folio 6r, le vieux Pandolfino explique «il molto leggere insegna bene vivere quando la discretione naturale non ha difecto », ce qui suggère, conformément à la pensée d'Aristote, que les lectures de philosophie morale ne sont utiles qu'à ceux qui sont déjà bien disposés. Or une partie de cette phrase est entre crochets et signalée par un trait dans la marge, à savoir le passage « io potrei poco narrare cose nuove a chi molto legge, pero che il molto leggere insegna ben vivere », d'où un contresens sur l'utilité de la lecture pour vivre vertueusement. C'est là un bon exemple de lecture partielle et donc fautive, mais qui illustre bien les attentes des lecteurs de la bibliothèque de Guadalajara.

${ }^{87}$ COVA, Luciano, Il Liber de virtutibus di Guido Vernani da Rimini: una rivisitazione trecentesca dell'etica tomista, Turnhout, Brepols, 2011, p. 261.
} 